Instituto Internacional de Investigación y Desarrollo Tecnológico Educativo INDTEC, C.A.

DOI: https://doi.org/10.29394/Scientific.issn.2542-2987.2019.4.13.5.90-113

OAI-PMH: http://www.indteca.com/ojs/index.php/Revista Scientific/oai

Artículo Original / Original Article

\title{
Una mirada al capital intelectual en universidades públicas
}

\author{
Autores: Javier José Castro Capitillo \\ Universidad Nacional Experimental "Rafael María Baralt", UNERMB \\ jccapitillo@hotmail.com \\ Zulia, Venezuela \\ https://orcid.org/0000-0002-3946-705X \\ Elide del Rosario Castellanos Santiago \\ Universidad Nacional Experimental "Rafael María Baralt", UNERMB \\ elidecastellanos255@hotmail.com \\ Trujillo, Venezuela \\ https://orcid.org/0000-0002-0301-0330 \\ Luz Marina Fonseca Rodríguez \\ Universidad Nacional Experimental "Rafael María Baralt", UNERMB \\ luzmfonseca72@hotmail.com \\ Zulia, Venezuela \\ https://orcid.org/0000-0002-4492-3769 \\ José Luís Lugo Barrios \\ Universidad Nacional Experimental "Rafael María Baralt", UNERMB \\ joseluislugo1@hotmail.com \\ Zulia, Venezuela \\ https://orcid.org/0000-0003-1315-9486
}

\section{Resumen}

La investigación estuvo orientada a analizar el capital intelectual desarrollado en la Universidad Nacional Experimental "Rafael María Baralt" (UNERMB), desde la perspectiva de sus componentes, considerando los aportes teóricos de Bueno, (2003); Suárez y Martín (2008); Navas y Ortiz (2002); Stewart (2001). Metodológicamente, el enfoque epistemológico es cuantitativo, tipo analítica, transeccional descriptivo, de campo, se aplicó un cuestionario de 34 ítems, con alternativas de respuestas múltiples (siempre, casi siempre, a veces, casi nunca y nunca). Para la validez, se aplicó la técnica de juicio de expertos y, para la confiabilidad se utilizó el coeficiente Alpha de Cronbach, arrojando un valor de 0.85 . La población censal fue de 42 docentes. Para procesar la data se utilizó estadística descriptiva. Los resultados reflejaron un alto nivel de desarrollo para cada componente del capital intelectual. Se concluyó que existe una alta aplicabilidad del capital intelectual dentro del entorno universitario, logrando considerar el capital humano, estructural, relacional en las funciones que ejecutan (docencia, investigación, extensión), considerando las actividades de su personal docente, así como los requerimientos planteados en la sociedad en relación a la formación de sus estudiantes. Por tanto, el capital intelectual es un activo importan para la efectividad de los procesos universitarios.

Palabras clave: capital humano; conocimiento; política laboral; productividad.

Fecha de Recepción: 30-10-2018
Fecha de Aceptación: 23-05-2019
Fecha de Publicación: 05-08-2019 


\title{
A look at the intellectual capital in public universities
}

\begin{abstract}
The research was oriented to analyze the intellectual capital developed in the National Experimental University "Rafael María Baralt" (UNERMB), from the perspective of its components, considering the theoretical contributions of Bueno, (2003); Suárez and Martín (2008); Navas and Ortiz (2002); Stewart (2001). Methodologically, the epistemological approach is quantitative, analytical type, descriptive transectional, field, a questionnaire of 34 items was applied, with alternative multiple answers (always, almost always, sometimes, almost never and never). For validity, the expert judgment technique was applied and, for reliability, Cronbach's Alpha coefficient was used, yielding a value of 0.85 . The census population was 42 teachers. To process the data, descriptive statistics were used. The results reflected a high level of development for each component of intellectual capital. It was concluded that there is a high applicability of intellectual capital within the university environment, managing to consider human capital, structural, relational in the functions they perform (teaching, research, extension), considering the activities of their teaching staff, as well as the requirements in society in relation to the training of its students. Therefore, intellectual capital is an important asset for the effectiveness of university processes.
\end{abstract}

Keywords: human capital; knowledge; labor policy; productivity.

Date Received: 30-10-2018
Date Acceptance: 23-05-2019
Date Publication:

05-08-2019 


\section{Introducción}

Como parte de la dinámica del entorno posmoderno, las organizaciones a nivel mundial, se desenvuelven en un contexto caracterizado por su complejidad y la necesidad de adaptarse al cambio competitivo, creativo, cambiante e innovativo. Para enfrentar ese escenario, es necesario desarrollar en los miembros de la organización, en cada uno de sus niveles las competencias necesarias para el cumplimiento efectivo de cada una de las actividades estratégicas y operativas, sobre la base de una planificación en materia de política laboral, enfrentándose a las nuevas exigencias del mercado, a los requerimientos de los consumidores, a las acciones gubernamentales, así como mediante el diseño de estrategias enmarcadas dentro de una concepción activa y participativa de cada integrante de la organización.

De acuerdo a lo anterior, se puede señalar que el personal de una organización representa el capital humano necesario para alcanzar los objetivos, específicamente los niveles de productividad. Esta posición, ha planteado la necesidad de conocer las expectativas reales o sentidas que influyen en su desempeño, logrando que se genere una actitud activa, un compromiso en las actividades que se realizan, por ello, es necesario que se considere al personal como un ser integral el cual requiere de atención por parte de la gerencia en sus carencias, así como limitaciones, dándole la posibilidad de desarrollarse desde la perspectiva personal como profesional.

Al respecto, el capital intelectual dentro de las universidades, cada día cobra mayor relevancia debido a que se considera como el único activo que proporciona las bases necesarias para sus operaciones a largo plazo, logrando brindar un servicio de calidad que satisfaga a corto, mediano y largo plazo las necesidades, tanto de los grupos de interés internos, representado por los miembros de la organización y, así como los externos, conformados por los clientes y la sociedad en general. 
Dentro del marco de esas ideas, las universidades como parte de la dinámica social, están sometidas a crecientes y complejas demandas que exigen una continua redefinición estratégica y operativa de sus funciones sociales, lo cual exige el replanteamiento y desarrollo continuo de las competencias de sus miembros, El talento humano, se convierte en la más importante herramienta de transformación y cambio y, de allí la necesidad de gestionarlo, de manera de crear condiciones idóneas para su desempeño y crecimiento.

Por tanto, en el presente estudio, se consideró como fuente de información a los profesores de la Universidad Nacional Experimental "Rafael María Baralt" (UNERMB), como unidad de estudio, dado que sus actividades se vinculan directamente con las funciones universitarias, y estas a su vez, se relacionan directamente con el capital humano, estructural y relacional, lo cuales integrados conforman el capital intelectual. En el marco de estas ideas, el estudio estuvo orientado a analizar el capital intelectual desarrollado en la mencionada institución, en el marco de cada uno de sus componentes.

Al respecto, dentro de una institución, el capital intelectual refiere el conocimiento intelectual, el componente intangible de la gestión organizacional, el cual está conformado por un conjunto de activos intangibles para desarrollar eficientemente a las organizaciones. En el marco de esas ideas, para Navas y Ortiz (2002): "del capital intelectual se suele reconocer al conjunto de activos intangibles con los que cuenta la organización" (pág. 163). En este sentido, para los autores citados se puede considerar al capital intelectual, en primer lugar, como una forma de creación de valor y, en segundo lugar, como un activo en su sentido tradicional.

\section{Contexto teórico}

\subsection{Capital humano}

Como componente del capital intelectual, representa el valor económico 
potencial, al constituirse en la mayor capacidad productiva de un individuo, pues refiere los conocimientos y experiencias de la población activa de un país u organización.

Es en ese sentido, la fuente de innovación y renovación, pues representa la plataforma intelectual sobre la cual sustentan los procesos estratégicos y operativos, que permiten vincular conocimientos, experiencias y vivencias, como mecanismos para elevar la calidad.

Plantea Stewart (2001a): que "el desarrollo de este componente exige de la reducción de los niveles de rutinización, como mecanismo para elevar la productividad laboral" (pág. 68); así como la creación continua de oportunidades de desarrollo, de manera de convertir el conocimiento tácito en explicito, con el objetivo de integrarlo a la base de conocimiento y, convertirlo en un mecanismo de inteligencia organizacional.

\subsubsection{Automotivación}

Según Maella (2010), la automotivación se puede definir como: "la disposición a poner un mayor esfuerzo para alcanzar un desempeño de calidad. Representa en este sentido, la parte visible del comportamiento que se constituye en una herramienta de modelado, de reforzamiento interno" (pág. 154). Esa condición de reforzamiento, se convierte en una herramienta importante para generar sentido de orientación y disposición plena para cumplir con las responsabilidades.

\subsubsection{Creatividad}

De acuerdo a Benegas (2015), la creatividad: "representa el ejercicio valorativo del potencial humano, la capacidad de inventiva e innovación, materializada en el mejoramiento continuo de los procesos" (pág. 75). La creatividad enlaza el potencial para visualizar oportunidades de mejora, de manera de articular nuevas y viejas ideas, para propiciar el cambio. 


\subsubsection{Experiencia}

Para Vargas (2005): la experiencia "se puede entender como una búsqueda del sujeto en la cual intenta reconstruir las notas realizadas de la onticidad (esto es, con notas reales)" (pág. 190). Una configuración valida de la interpretación que ha previsto (categórica, conceptual o nocionalmente) sobre el ser. La experiencia es un objeto válido de conocimiento que revela la estructura, o al menos parte de la estructura, de la actividad cognitiva del individuo, por ello su conocimiento es fundamental.

\subsubsection{Productividad}

Con relación a la productividad laboral, apunta Fernández (2010): que "cuando una organización desea exige a sus miembros el cumplimiento de sus responsabilidades con niveles adecuados de calidad y excelencia, de manera que se incremente considerablemente la productividad de sus miembros" (pág. 76); debe invertir en los procesos de formación y, en el desarrollo de condiciones que contribuyan a elevar el desempeño, satisfacción y niveles motivacionales de sus miembros.

\subsection{Capital estructural}

De acuerdo a Stewart (2001b), en relación al capital estructural: "refiere el conjunto de sistemas requeridos para generar, procesar y compartir conocimientos, sistematizándolos en plataforma estructurales que se constituyen en la base de datos que debe sustentar la gestión del conocimiento" (pág. 85).

Esta plataforma estructural, hace énfasis en los factores tecnológicos, así como la infraestructura de la organización, lo cual le proporcionan a nivel estructural, las rutinas, procedimientos, sistemas de bases de datos, lo cual representa el conocimiento explícito, traducido en propiedad intelectual. 


\subsubsection{Procedimientos}

Para Suárez y Martín (2008a): "conforme la gestión de las PYME se profesionaliza... las organizaciones formalizan sus procedimientos" (pág. 234). En este sentido, un procedimiento, refiere el conjunto de pasos $u$ operaciones que permiten cumplir con las actividades propias de un proceso operativo. El término, alude desde la etimología del mismo, a la acción, pues constituye de acuerdo a su raíz, procede, a la acción que orienta la forma de actuar de una forma determinada para alcanzar un propósito. En términos generales, el procedimiento refiere el conjunto de pasos o acciones las cuales conducen al desarrollo de las actividades de un proceso, en términos de eficiencia.

\subsubsection{Automatización de los procesos}

El desarrollo tecnológico, ha permitido de acuerdo con Suárez y Martín (2008b): "competir en los mercados internacionales, que ha conllevado una renovación institucional y, con ello, un cambio en la actitud de los empresarios que buscan adaptarse y subsistir, en algunos casos ingresar a una dinámica globalizadora" (pág. 237). Al respecto, la modificación de los procesos tradicionales, desde el trabajo manual, hacia la tecnificación de las operaciones y, el control de los procesos a través de los sistemas de cómputo, lo cual refiere la automatización de los procesos.

\subsubsection{Autogestión}

De acuerdo a Suárez y Martín (2008c): "si una empresa puede hacer que su producto o servicio sea especialmente atractivo, sus posibilidades se ampliarán de manera considerable" (pág. 235). Lo anterior exige, un conjunto de prácticas administrativas de autogestión, en un contexto social, las cuales se desarrollan por su condición, en un clima de democracia participativa, donde el colectivo, mantiene el autocontrol de los procesos. 


\subsubsection{Canales de comunicación}

Para Kotler y Keller (2012), establecen que el canal de comunicación: "son los medios por los cuales las empresas intentan informar, persuadir y recordar a los consumidores, de manera directa o indirecta" (pág. 476); un mensaje a su público de interés. Los canales de acuerdo a los autores citados más utilizados son los medios escritos, así como el conjunto de mecanismos y recursos audiovisuales utilizados en la actualidad, en la época de la llamada sociedad de la información y de la comunicación, en la cual los sistemas digitales han permitido masificar las redes de información eliminando las barreras de tiempo y espacio.

\subsubsection{Estructura organizacional}

Para Suárez y Martín (2008d): "es importante reconocer que el capital humano... potencia su capacidad en una organización cuando la estructura social y el proceso organizacional se articula a otros recursos" (pág. 232); al respecto, la estructura de la organización representa la red de relaciones y jerarquías dentro de las cuales se contextualiza el quehacer, refiriendo los niveles funcionales, la red de relaciones, la ubicación de las funciones y el manejo del poder, en el marco de la red de relaciones y poder propias de cada tipo de estructura.

\subsection{Capital relacional}

Otro componente del capital intelectual, está referido de acuerdo a Stewart (2001c): "al capital relacional, relacionado específicamente con la relación cliente-empresa" (pág. 113). Refiere, el valor de las relaciones que una organización mantiene con sus grupos de interés o clientes externos e involucra la gestión efectiva de las bases de datos, la capacidad de crear oportunidades de mercado y cliente potenciales, de manera de diversificar la cartera de cliente dentro de una red que integren accionistas, proveedores, 
gobierno, sociedad, entre otros.

\subsubsection{Grado de cooperación}

En este sentido, hablar de la cooperación es elemental para la subsistencia en sociedad, debido a que es una manera eficaz y más eficiente de gestionar, canalizar y alcanzar los propósitos tantos en función de un beneficio colectivo. Como parte de la dinámica organizacional la cooperación sustenta el trabajo de equipo e involucra en esencia la condición de apoyo que debe sustentar e integrar los esfuerzos del colectivo de la organización. Al respecto, Bueno (2003a): "se requiere la evaluación de los aspectos claves que mantienen y cohesionan la estructura de la organización y permiten que las actividades se desarrollen de forma sistemática y coherente" (pág. 29). En este orden de ideas, cuando el grado de cooperación aumenta, se fortalece y mejora el trabajo de equipo, generándose lazos de apoyo que contribuyen a la eficiencia de las operaciones.

\subsubsection{Vinculación con la sociedad}

La vinculación está relacionada con la integración de esfuerzos y el desarrollo de un espíritu del compromiso social, orientado a la satisfacción de las necesidades del grupo social en el cual interactúa la organización, según Bueno (2003b): "en la economía interconectada actual, resulta necesario contrastar los resultados de los indicadores con las mediciones de otras empresas que se encuentren dentro del mismo sector, para intentar normalizar las áreas de evaluación" (pág. 33). Las organizaciones en este orden de ideas, al plantearse una relación ecológica con la sociedad, asumen una responsabilidad social, lo cual plantea la necesidad de vincularse a ella, para conocer sus requerimientos y satisfacer las necesidades a corto, mediano y largo plazo. 


\subsubsection{Vinculación de los egresados}

Los mecanismos de vinculación institucional, generan diversas formas de interacción con su entorno y su interioridad, para el caso de las Universidades. Al respecto, para Inche y Chung (2004): "es el valor de la base de clientes, de la capacidad de mantener la relación con ellos y de su potencial, y el valor referido a otros agentes del entorno como los proveedores, las autoridades, etc." (pág. 43).

\subsubsection{Grado de satisfacción de los participantes}

A juicio de Koontz (2013), la satisfacción del cliente refiere: "el mecanismo idóneo para valorar la calidad de los procesos y, está relacionado con la capacidad de un ente, proceso o unidad para cumplir con las expectativas y necesidades" (pág. 261). En ese orden de ideas, la satisfacción del cliente resulta ser una de las metas a las cuales cualquier organización aspira, para poder sobrevivir y desarrollar ventajas competitivas, para alcanzar de esa forma el éxito.

\subsubsection{Innovación}

Para Oberto (2007), la innovación: "relaciona la creatividad como medio de transformación mejoramiento de los procesos, e involucra la generación de nuevas ideas, e integración de ideas existentes" (pág. 45); dentro de un continuo que propicia el mejoramiento y la perfección de las técnicas, pasos, procedimientos e instrumentos necesarios para cumplir con las operaciones.

\section{Metodología (Materiales y métodos)}

La visión del mundo requiere una ubicación epistemológica de los investigadores, de tal manera que las decisiones derivadas de los procedimientos metódicos sean consecuentes y coherentes con la posición que este asume. Desde esta perspectiva y sobre la base de los objetivos que 
sustentaron el estudio investigativo, el mismo se ubicó en el enfoque empíricoanalítico-positivista, el cual estuvo sustentado en el manejo cuantitativo del dato, derivado de su procesamiento estadístico.

Por otra parte, de acuerdo al objetivo planteado, la investigación se tipifica como analítica, apoyada en un diseño no experimental, transeccional descriptivo y de campo. En este sentido, para la recolección de datos, fueron consideradas como fuentes de información a los 42 docentes que asumen funciones de jefes de cátedras en los diferentes programas de la UNERMB, utilizando para la aplicación de los cuestionarios el criterio de censo poblacional, pues se asumió como muestra a la totalidad de la población, a la cual se le aplicó como instrumento de recolección de datos, un cuestionario estructurado conformado por 34 ítems y alternativas de respuesta múltiple (Siempre, casi siempre, a veces, casi nunca y nunca).

Para aplicar el instrumento, fue necesario determinar su validez a través de un juicio de expertos y, el coeficiente Alpha de Cronbach, el cual arrojó un valor de 0.85 , para determinar su confiabilidad. En cuanto al procesamiento de los datos, derivado del enfoque cuantitativo que sustentó la investigación, el mismo se realizó a través del método de estadística descriptiva, utilizando medidas de tendencia central (promedio) y, variabilidad (desviación estándar), valores que fueron interpretados a través de los siguientes baremos, presentados en los cuadros 1 y 2.

En el caso del promedio, se establecieron 5 rangos, intervalos y categorías, cuyos valores oscilan entre 1 y 5 , vinculados en este caso con el valor asignado a las categorías de respuestas. En el cuadro 1, se describe en este sentido el baremo para la interpretación de la media o promedio aritmético: 
Cuadro 1. Baremo para la interpretación del promedio aritmético.

\begin{tabular}{|c|c|c|}
\hline Rango & Intervalo & Categoría \\
\hline 1 & $4.21-5$ & Muy Alto Desarrollo \\
\hline 2 & $3.41-4.20$ & Alto Desarrollo \\
\hline 3 & $2.61-3.40$ & Moderado Desarrollo \\
\hline 4 & $1.81-2.60$ & Bajo Desarrollo \\
\hline 5 & $1-1.80$ & Muy Bajo Desarrollo \\
\hline
\end{tabular}

Fuente: Los Autores (2018).

Por otra parte, con respecto a la desviación estándar, medida que refleja el nivel de dispersión, los intervalos oscilan entre 0 y 4 , valores que representan la más alta y baja dispersión. Al respecto, en el cuadro 2, se detalla el baremo para la interpretación de la desviación, dentro del cual es importante considerar, que la dispersión de las respuestas es inversa a la confiabilidad, en el sentido, que una dispersión alta, refiere una baja confiabilidad y, viceversa.

Cuadro 2. Baremo para la interpretación de la desviación estándar

\begin{tabular}{|c|c|c|}
\hline Rango & Intervalo & Categoría \\
\hline 1 & $3.21-4$ & Muy Alta Dispersión \\
\hline 2 & $2.41-3.20$ & Alta Dispersión \\
\hline 3 & $1.61-2.40$ & Moderada Dispersión \\
\hline 4 & $0.81-1.60$ & Baja Dispersión \\
\hline 5 & $0-0.80$ & Muy Baja Dispersión \\
\hline
\end{tabular}

Fuente: Los Autores (2018).

\section{Resultados (análisis e interpretación de los resultados)}

Tabla 1. Variable: Capital intelectual

\begin{tabular}{|l|r|c|}
\hline \multicolumn{1}{|c|}{ Dimensiones } & Promedio & Desviación \\
\hline Capital humano & 3,59 & $\mathbf{0 , 3 9}$ \\
\hline Capital estructural & 3,51 & $\mathbf{0 , 3 5}$ \\
\hline Capital relacional & 3,27 & $\mathbf{0 , 4 5}$ \\
\hline \multicolumn{1}{|c|}{ Total general } & $\mathbf{3 , 4 6}$ & $\mathbf{0 , 4 0}$ \\
\hline
\end{tabular}

Fuente: Los Autores (2018). 
Como se observa en la tabla 1, para la Variable Capital intelectual, el procesamiento arrojó un promedio de 3.46, con una muy baja dispersión de 0.40 , ubicándose en la categoría alta, al indicar que con esa frecuencia se consideran dentro de la universidad y específicamente en sus funciones el capital humano, estructural y relacional, el cual apoya las actividades vinculadas de acuerdo al reglamento general de la universidades con sus funciones, a saber la docencia, investigación y extensión, logrando cumplir cada una de las actividades sobre la base de los lineamientos institucionales, logrando así realizar investigaciones para el servicio de la comunidad; formar profesionales considerando los requerimientos del mercado laboral, y ser responsables socialmente mediante actividades de extensión.

Tabla 2. Dimensión: Capital humano

\begin{tabular}{|c|c|c|}
\hline Indicadores & Promedio & Desviación \\
\hline Automotivación & 3,85 & 0,41 \\
\hline Creatividad & 2,98 & 0,31 \\
\hline Experiencia & 3,44 & 0,35 \\
\hline Productividad & 3,48 & 0,36 \\
\hline Total general & 3,59 & 0,39 \\
\hline
\end{tabular}

Fuente: Los Autores (2018).

En cuanto a la dimensión capital humano, presentada en la tala 2, el procesamiento de los datos arrojó un de 3.59, con una muy baja dispersión de 0.39; ubicándose en la categoría alta, al señalar que con esa frecuencia se fomenta en la institución; la automotivación, productividad, así como experiencia, realizando sus actividades mediante el apoyo brindando por la universidad. Sin embargo, se visualiza un nivel moderado en cuanto a la aplicación de la creatividad en sus funciones como docente.

En cuanto al indicador automotivación, como se observa en la tabla, el promedio fue de 3.85 y, la dispersión de 0.41 ; indicando una categoría alta, con una muy baja dispersión de las respuestas, lo cual refiere una muy alta 
confiabilidad de las mismas, al afirmar los docentes que con una alta frecuencia se identifica con las actividades que realiza en la institución; asimismo, las actividades que realiza le generan una satisfacción personal.

En lo que respecta al indicador creatividad, el promedio fue de 2.98, con una muy baja dispersión de 0.31; ubicándose en la categoría moderada, pues de acuerdo a las respuestas de los profesores, con una moderada frecuencia se emplean herramientas creativas para lograr los objetivos en su entorno laboral; asimismo, se aplica la imaginación para resolver situaciones imprevistas.

En lo que respecta al indicador experiencia, el promedio fue de 3.44, con una muy baja dispersión de 0.35 ; al indicar que con moderado nivel se aprovechan los conocimientos adquiridos durante sus años de servicio en cada una de las actividades que ejecuta en la universidad; además, se le considera su experiencia en las nuevas actividades que realiza la universidad.

En cuanto al indicador productividad, el promedio fue de 3.48, con una dispersión de 0.36; indicando una categoría alta, con una muy baja dispersión, al señalar los profesores que las actividades que realizan generan los resultados esperados; asimismo, en sus funciones realizan sus actividades utilizando los recursos de manera racional.

Tabla 3. Dimensión: Capital estructural

\begin{tabular}{|l|c|c|}
\hline \multicolumn{1}{|c|}{ Indicadores } & Promedio & Desviación \\
\hline Procedimientos & 2,96 & $\mathbf{0 , 3 2}$ \\
\hline Automatización de los procesos & 2,36 & $\mathbf{0 , 3 6}$ \\
\hline Autogestión & 4,15 & $\mathbf{0 , 3 4}$ \\
\hline Canales de comunicación & 3,52 & $\mathbf{0 , 3 8}$ \\
\hline Estructura organizacional & 4,54 & $\mathbf{0 , 3 7}$ \\
\hline \hline \multicolumn{1}{|c|}{ Total general } & $\mathbf{3 , 5 1}$ & $\mathbf{0 , 3 5}$ \\
\hline
\end{tabular}

Fuente: Los Autores (2018).

En lo que se refiere a la dimensión capital estructural, presentada en la 
tabla 3, el procesamiento de los datos arrojó un promedio de 3.51, con dispersión de 0.35 , lo cual indica que desde la perspectiva de las respuestas de los docentes un nivel alto, en relación a la aplicación de la autogestión, la utilización de canales de comunicación, así como el respeto de la estructura organizacional, sin embargo, se visualiza un nivel bajo en cuanto al indicador automatización de los procesos.

En cuanto al indicador procedimientos, el procesamiento de las respuestas de los docentes, arrojó un promedio de 2.96 con una desviación de 0.32; indicando que con una frecuencia moderada; consideran los procedimientos institucionales para el desarrollo de sus actividades; asimismo, se cumplen a cabalidad cada uno de los procedimientos que permitan lograr los objetivos, la desviación en este sentido, se ubicó en una categoría muy baja, lo cual indica una alta confiabilidad de las respuestas.

Para el indicador automatización de los procesos, el promedio alcanzado en el procesamiento de los datos fue de 2.36, con una muy baja dispersión de 0.36; ubicándose en la categoría baja; al señalar que con baja frecuencia se utiliza la tecnología para realizar las actividades institucionales; asimismo, se optimiza el tiempo mediante la aplicación de la tecnología en las actividades que ejecuta, situación que refleja una debilidad importante en cuanto al uso de esta condición.

En lo que respecta al indicador autogestión, los datos procesados arrojaron un valor de 4.15, con una muy baja dispersión de 0.34 ; indicando una categoría alta, al responder que con esa frecuencia utilizan sus recursos para realizar las actividades institucionales, aunque con las limitaciones inherentes a su presupuesto.

El indicador canales de comunicación, el procesamiento arrojó un promedio de 3.52 , con una muy baja dispersión de 0.38 ; indicando una categoría muy alta; al responder con esa frecuencia, la información fluye en todos los niveles institucionales; además, aplican canales de comunicación 
formal para proporcionar información clara.

En relación al indicador estructura organizacional, se observa en la tabla un promedio de 4.54, con una muy baja dispersión de 0.3 , pues los docentes señalaron que una muy alta frecuencia se reconoce la estructura organizacional establecida en la universidad; asimismo, se les asignan actividades considerando su posición en la misma, lo cual facilita el desempeño de sus funciones.

Tabla 4. Dimensión: Capital relacional

\begin{tabular}{|l|c|c|}
\hline \multicolumn{1}{|c|}{ Indicadores } & Promedio & Desviación \\
\hline Grado de cooperación & 4,11 & $\mathbf{0 , 4 8}$ \\
\hline Vinculación con la sociedad & 3,98 & $\mathbf{0 , 4 7}$ \\
\hline Vinculación de los egresados & 2,36 & $\mathbf{0 , 4 3}$ \\
\hline Grado de satisfacción de los participantes & 3,52 & $\mathbf{0 , 4 4}$ \\
\hline Innovación & 2,39 & $\mathbf{0 , 4 1}$ \\
\hline \hline \multicolumn{1}{|c|}{ Total general } & $\mathbf{3 , 2 7}$ & $\mathbf{0 , 4 5}$ \\
\hline
\end{tabular}

Fuente: Los Autores (2018).

Con respecto a la dimensión capital relacional, como se observa en la tabla 4, el promedio alcanzado fue de 3.27 y una desviación de 0.45, resultados que se ubican de acuerdo a los niveles en un categoría alta y, una muy baja dispersión, al señalar los profesores una baja aplicación de la vinculación con los egresados, innovación, sin embargo, con una alta aplicabilidad con el grado de cooperación, vinculación con la sociedad, grado de satisfacción estudiantil, reflejando deficiencias en las relaciones con el sector externo, el cual es fundamental mantener contacto directo con la sociedad para lograr los objetivos planteados.

En cuanto al indicador grado de cooperación, el promedio alcanzado fue de 4.11, con una desviación de 0.48; al indicar los docentes que con una frecuencia alta apoyan a sus compañeros para alcanzar los objetivos planteados; asimismo, reconoce el esfuerzo individual sobre la base de su 
trabajo en equipo.

Para el indicador vinculación con la sociedad, el procesamiento de los datos arrojó un promedio de 3.98, con una muy baja dispersión de 0.47 , indicando que con una alta frecuencia se realiza acciones de responsabilidad social para lograr la función de extensión, asimismo, las funciones universitarias permiten un vínculo con la sociedad.

En lo que respecta al indicador vinculación de los egresados, el promedio obtenido a través del procesamiento de los datos fue de 2.36, con una muy baja dispersión de 0.43; ubicándose en la categoría de baja frecuencia al indicar los profesores en sus respuestas que no se apoya a los egresados en actividades relacionadas con su casa de estudio; no se promueve la actualización profesional de los egresados.

El indicador grado de satisfacción de los participantes, el promedio alcanzado fue 3.52, con una muy baja dispersión de 0.44; al indicar los encuestados con una frecuencia baja, se realizan actividades que contribuyen a satisfacer las funciones de la universidad; asimismo, su esfuerzo en las actividades que realiza contribuye a la satisfacción de las necesidades institucionales.

Para el indicador innovación, se observa en la tabla 4, que el promedio obtenido fue de 2.39, con una muy baja dispersión de 0.41 , indicando que con una frecuencia baja se actualizan sus actividades sobre la base de herramientas innovadoras; no consideran que la innovación sea una herramienta que permite alcanzar los objetivos institucionales.

\section{Conclusiones}

Derivado del análisis e interpretación de los resultados, se refleja una alta aplicación de la automotivación; productividad y experiencia, logrando brindarle el apoyo necesario a su personal en la ejecución de sus funciones, sin embargo, se refleja un nivel moderado en cuanto a la creatividad, lo cual 
es fundamental para lograr que sus actividades sean ejecutadas con mayor firmeza.

Se evidenció además, un nivel alto en la aplicación y utilización de los canales de comunicación, de la estructura organizativa, así como la automatización de los procesos, autogestión, logrando que las actividades se cumplan de acuerdo a lo establecido por la universidad, sin embargo, existe una baja aplicación en la autogestión y los procedimientos, los cuales deben contribuir en aprovechar el tiempo, así como los recursos de la institución.

Por otra parte, se evidenció, alto nivel en la aplicabilidad del grado de cooperación, vinculación con la sociedad, así como el grado de satisfacción de los grupos de interés tanto internos como externos; sin embargo, con un nivel bajo la vinculación con los egresados y la innovación, afectando en cierta medida la función de extensión y, de forma directa, el nivel de pertinencia académica alcanzado.

\section{Recomendaciones}

Los resultados y conclusiones, permitieron plantear las siguientes recomendaciones: Proporcionar mayor apoyo a los docentes para el despliegue efectivo de su función académica, en el marco de sus roles como docente, investigador y promotor social, dado que las actividades que realizan son por los propios medios, sin un aporte por parte de la universidad para realizar investigaciones, así como actividades sociales.

Realizar estrategias que permitan involucrar a los egresados dentro de las actividades institucionales, lo cual contribuye con el desarrollo de la universidad, así como de la sociedad en general, debido a que es pertinente darles un seguimiento a los profesionales que se forman, asimismo, darle nuevas herramientas que permitan actualizar sus competencias.

Apoyar a los docentes en la automatización de ciertos procesos, que permita que sus funciones se ajusten a las nuevas herramientas tecnológicas, 
logrando una mayor interacción con el estudiante, así como con su entorno.

El docente debe aplicar estrategias creativas y de innovación, logrando que las actividades que realicen puedan ser aprovechadas en su totalidad, para ello, es pertinente que utilice su formación, imaginación en su quehacer docente.

\section{Referencias}

Benegas, A. (2015). La Libertad Es Respeto Reciproco. ISBN: 1505695775. Washington D.C., Estados Unidos: Cato Institute.

Bueno, E. (2003a,b). Metodología para la elaboración de indicadores de capital intelectual. Documentos Intellectus, nro 4, 1ra. Edición, eISSN: 1578-911X. Madrid, España: DICREA, Creaciones Gráficas, S.L. Recuperado de:

https://www.researchgate.net/publication/298346311 Metodologia par a elaboracion de indicadores de capital intelectual

Fernández, R. (2010). La productividad y el riesgo psicosocial o derivado de la organización del trabajo. ISBN: 978-84-9948-414-3; ISBN: 97884-9948-146-3. España: Editorial Club Universitario.

Inche, J., \& Chung, A. (2004). Indicadores de Gestión del Conocimiento en la Facultad de Ingeniería Industrial. Industrial Data, 7(2), 41-45, eISSN: 1560-9146. Recuperado de:

http://www.redalyc.org/articulo.oa?id=81670207

Koontz, T. (2013). Social learning in collaborative watershed planning: the importance of process control and efficacy. Journal of Environmental Planning and Management, 57(10), 1572-1593, e-ISSN: 1360-0559. Recuperado de:

https://doi.org/10.1080/09640568.2013.820658

Kotler, P., \& Keller, K. (2012). Dirección De Marketing. 14ª edición, ISBN: 978-607-32-1245-8. México: Pearson Educación de México de C.V. 
Maella, P. (2010). Las Variables y las Conductas de la Eficacia Personal. Occasional Paper, OP-174. Barcelona, España: IESE Business School - Universidad de Navarra. Recuperado de: https://media.iese.edu/research/pdfs/OP-0174.pdf

Navas, J., \& Ortiz, M. (2002). El capital intelectual en la empresa. Análisis de criterios y clasificación multidimensional. Economía Industrial, 4(346), 163-171. Recuperado de:

https://www.mincotur.gob.es/Publicaciones/Publicacionesperiodicas/Ec onomialndustrial/RevistaEconomialndustrial/346/14\%20EMILIO\%20N AVAS.pdf

Oberto, A. (2007). Conocimientos tecnológicos e innovación en el sector de termoplásticos del estado Zulia. Colección Textos Universitarios, ISBN: 9806992547 . Mérida, Venezuela: Universidad del Zulia, 97 págs. Recuperado de:

http://catalogo.luz.edu.ve/cgi-bin/koha/opacdetail.pl?biblionumber $=18981$

Suárez, T., \& Martín, M. (2008a,b,c,d). Impacto de los capitales humano y organizacional en las estrategias de la pyme. Cuadernos de Administración, 21(35), 229-248, e-ISSN: 1900-7205 (En línea); ISSN: 0120-3592 (Impreso). Recuperado de:

https://revistas.javeriana.edu.co/index.php/cuadernos admon/article/vi $\underline{\mathrm{ew} / 4006}$

Stewart, T. (2001a,b,c). La Nueva Riqueza de las Organizaciones: EI Capital Intelectual. ISBN 10: 9506412537 ; ISBN 13: 9789506412531. Buenos Aires, Argentina: Gránica Adelphi, 376 págs.

Vargas, G. (2005). La experiencia de ser: Tratado de metafísica. 1ra Edición, ISBN: 958-692-361-4. Bogotá, Colombia: Impresor Sociedad de San Pablo. 


\section{Javier José Castro Capitillo}

e-mail: jccapitillo@hotmail.com

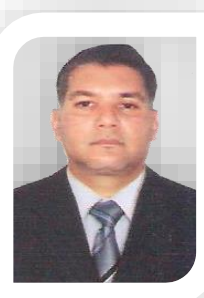

Nacido en Cabimas, Estado Zulia, Venezuela, el 10 de abril del año 1973. Licenciado en Administración, Mención Gerencia Industrial en el año 1999 en la Universidad Nacional Experimental "Rafael María Baralt" (UNERMB); Magister Scientiarum en Gerencia de Recursos Humanos (UNERM, 2010);

Especialización de Docencia Universitaria en el año 2012; y Doctor en Ciencias Gerenciales en el año 2016 en la Universidad "Dr. Rafael Belloso Chacín"; Formación Docente para Egresados - Componente Docente (UNERMB, 2017). Actualmente me desempeño en la UNERMB, como Docente de las Materias: Gerencia de Mercado, Taller de Acción Profesional I y II, Matemáticas II, Estrategias Gerencial. Finanzas de la Empresa, Fundamentos Matemáticos y Aritmética I. 


\section{Elide del Rosario Castellanos Santiago}

e-mail: elidecastellanos25@hotmail.com

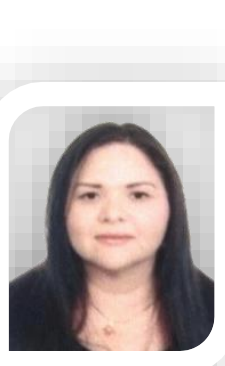

Nacida en Valera, estado Trujillo, Venezuela, el 2 de julio del año 1975. Lcda. en Educación Integral; MSc. en Docencia para Educación Superior; Dra. en Educación. Docente en el Ministerio del Poder Popular para la Educación, N.E.R. 233; Profesora en la Misión Sucre Aldea Universitaria "Cruz Carrillo"; Profesora Asesora del Programa Nacional de Gestión Social, en Sabana de Mendoza. 


\section{Luz Marina Fonseca Rodríguez \\ e-mail: luzmfonseca77@hotmail.com}

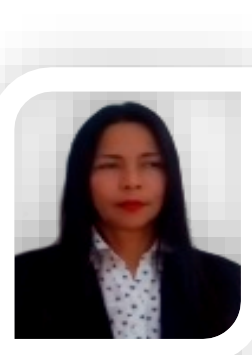

Nacida en Cabimas, estado Zulia, Venezuela, el 4 de febrero del año 1972. Lcda. en Administración, Mención Gerencia Industrial; MSc. en Gerencia de Recursos Humanos; Doctorante en Educación. Personal Administrativo (contratada); Jefe de la Unidad de Almacén en el Instituto Universitario de

Tecnología Cabimas. 
José Luís Lugo Barrios

e-mail: joseluislugo@hotmail.com

Nacido en Cabimas, estado Zulia, Venezuela, el 8 de

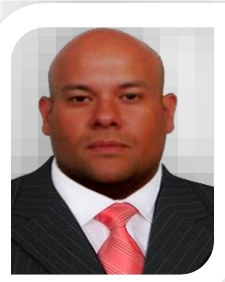
marzo del año 1981. Ing. Mecánico; MSc. en Gerencia de Empresas, Mención Gerencia de Operaciones. Doctorante en Educación. Docente Contratado con categoría de Instructor a Tiempo Completo en el Instituto Universitario de Tecnología Cabimas. 